\title{
Does syncope require rhythmic and non-rhythmic evaluation in patients with previous MI?
}

\author{
B Brembilla-Perrot, C Suty-Selton, ${ }^{1} \mathrm{~F}$ Alla, ${ }^{2}$ PY Zinzius, ${ }^{1}$ H Blangy, ${ }^{1}$ B Azman, ${ }^{1}$ \\ A Terrier de la Chaise, ${ }^{1}$ P Louis, ${ }^{1}$ L Groben, ${ }^{1} \mathrm{~K}$ Djaballah, ${ }^{1} \mathrm{O}$ Selton, ${ }^{1} \mathrm{~S}$ Magalhaes, \\ L Muresan, ${ }^{1} \mathrm{~J}$ Cedano, ${ }^{1}$ A Abdelaal, ${ }^{1}$ N Sadoul ${ }^{1}$
}

${ }^{1}$ Cardiology, $\mathrm{CHU}$ of Brabois, Vandœuvre-lès-Nancy, France ${ }^{2}$ Epidemiology, CHU, Nancy, France

\section{Correspondence to}

Dr B Brembilla-Perrot, Cardiologie, CHU de Brabois, Vandœuvre-lès-Nancy 54500, France; b.brembilla-perrot@ chu-nancy.fr

Accepted 11 May 2010

\begin{abstract}
Background Multiple factors, in addition to left ventricular ejection fraction (LVEF) influence the risk of mortality in coronary artery disease. The purpose of this study was to evaluate the main causes of syncope after myocardial infarction (MI) and to propose an algorithm of management.
\end{abstract}

Methods 356 patients consecutively admitted for syncope and history of $\mathrm{MI}$ (>1 month), without ventricular tachycardia (VT), underwent echocardiography, Holter monitoring, head-up tilt test, exercise testing, signal-averaged ECG,

electrophysiological study (EPS) and evaluation of coronary status. The mean follow-up was $4 \pm 2$ years.

Results Monomorphic VT, ventricular flutter or fibrillation (VF) and supraventricular tachyarrhythmia were respectively induced at EPS in 87, 63 and 39 patients; conduction disturbances were noted in 23 patients, and 57 patients had several abnormalities. Among the 144 patients with negative EPS, coronary ischaemia was identified in 37 patients, and hypervagotonia in 27 patients. All studies remain negative in 84 patients $(23.6 \%)$, more frequently women $(p<0.001)$. Four patients died suddenly during follow-up. A longer QRS duration, a lower LVEF and grade IVa,b of Lown on Holter ECG were associated with the induction of VT.

$\mathrm{LVEF}<40 \%$ and VT/NF induction were predictors of cardiac mortality, VT was a predictor of sudden death, and low LVEF and advanced age were predictors of death by heart failure.

Conclusion Myocardial ischaemia, hypervagotonia, conduction abnormalities, ventricular or supraventricular tachyarrhythmias were identified in $76 \%$ of patients with syncope after MI. Several factors of syncope were found in 57 patients (16\%). Non-invasive rhythmological and systematic coronary status assessment should be recommended in patients with syncope following MI.

Patients with syncope and history of myocardial infarction are at risk of sudden death. In the era of implantable cardioverter defibrillator (ICD), ${ }^{1}$ the left ventricular ejection fraction (LVEF) is probably a more important factor of mortality than syncope. However, causes for syncope following MI are multiple, ${ }^{2}$ and ICD implantation does not resolve all causes of syncope and cardiac death. ${ }^{3}$ Electrophysiological study (EPS) was widely used several years ago, ${ }^{4}$ but was considered without interest for the risk stratification in coronary heart disease. ${ }^{6}$ However, inducible VT remains an important and independent factor of cardiac mortality. ${ }^{7}$

The assessment of patients who present with a syncope following MI may be debatable. In some cases, it is limited to the evaluation of LVEF, and ICD is indicated if LVEF is lower than $36 \% .{ }^{1}$ However, the proarrhythmic effect of ICD and other complications are well known, ${ }^{8}$ and for others this justifies a specific search of the cause of syncope with an adapted therapy. Among patients with LVEF higher than $35 \%$, the implantation of a loop recorder is indicated $^{9-11}$ until the recurrence of syncope with a risk of trauma or sudden death, if syncope recurs.

The purpose of the study was to evaluate the factors responsible for syncope and the risk of recurrence after a treatment guided by this evaluation. We then constructed an algorithm to manage the patients with syncope and history of myocardial infarction.

\section{POPULATION OF STUDY}

All patients with a history of myocardial infarction, who presented with at least one episode of unexplained syncope, admitted for electrophysiological study were systematically recruited over a 15-year period. Unexplained syncope was defined as a short loss of consciousness, without any obvious aetiology. Obvious aetiology included paroxysmal second- or third-degree AV block, sustained supraventricular or ventricular arrhythmia, and vasovagal syncope induced by cough, miction or abdominal pain. ${ }^{5}$ Patients were excluded if they had unstable angina, recent acute myocardial infarction $(<1$ month), recent coronary angioplasty or coronary bypasses surgery ( $<6$ weeks), if they were in NYHA III-IV stage, had end-stage non-cardiac disease or received chronic amiodarone treatment. Patients with drug-induced bradycardia or tachycardia (accounting for around 10\% of syncope) were also excluded; they represent about $10 \%$ of admissions for syncope. Patients with effort-related syncope and obviously ischaemic syncope were excluded, because an electrophysiological study was not performed in these patients.

During the period of study, 371 patients were recruited, of whom 15 were excluded for drugrelated syncope and obviously ischaemic-related syncope. Three additional patients were excluded because they were lost on follow-up.

The remaining 356 patients, aged 31 to 85 years (mean age 66士11; 56 women and 300 men), represent the study population.

\section{METHODS}

Patients underwent several investigations in the absence of antiarrhythmic drugs after giving informed consent. Personal and familial clinical history, list of drugs taken at the time of syncope 
and clinical examination were initially noted. Most patients received ACE inhibitors. Beta-blockers and digoxin were stopped before EPS.

The following non-invasive studies were performed: (1) surface ECG; (2) $24 \mathrm{~h}$ Holter monitoring (Elatec); (3) thallium exercise scintigraphy or exercise testing in 240 patients able to perform the exercise; (4) LVEF at the time of investigations for syncope determined by $2 \mathrm{D}$ echocardiography and/or left ventricular angiography; (5) measurement of ORS duration at signal-averaged ECG; and (6) head-up tilt test without provocative drugs in 110 patients with negative EPS or inducible ventricular flutter or fibrillation.

The following invasive studies were performed: (1) right and left angiography and coronary angiography was indicated in 301 patients; (2) complete EPS according to a protocol previously reported $^{12}$ was systematic. The protocol included assessment of sinoatrial conduction function and atrioventricular conduction. Programmed atrial stimulation was performed with up to two extrastimuli and then programmed right ventricular stimulation up to three extrastimuli at the right ventricular apex and right ventricular outflow tract. Short coupling intervals ( $<200 \mathrm{~ms})$ were not used in our study. If the study remained negative, the protocol was repeated after 2 to $4 \mu \mathrm{g} / \mathrm{min}$ isoproterenol infusion. Arterial blood pressure was continuously monitored by an external sphygmomanometer (Baxter, Hayashikomaki, Japan). A carotid sinus massage was performed in the supine position, except in patients with a known carotid atheroma.

\section{Definitions}

Inducible supraventricular tachyarrhythmia was assumed to be related to syncope if it was sustained, that is, lasting at least $3 \mathrm{~min}$, either spontaneously terminating but reproducible or permanent, associated with a decline in systolic arterial blood pressure of at least $30 \%$ and with symptoms similar to spontaneous dizziness.

Induced ventricular tachyarrhythmias were categorised as monomorphic VT $(<270 \mathrm{bpm})$, ventricular flutter ( $>270 \mathrm{bpm})$ or ventricular fibrillation $(\mathrm{VF})$.

Abnormal electrophysiological findings were categorised as sinus node dysfunction, conduction disturbances, hypervagotonia, inducible supraventricular tachyarrhythmia or inducible ventricular tachyarrhythmia (VT/NF) according to classical diagnostic criteria. ${ }^{12}$ When several anomalies were noted, including the induction of a ventricular tachyarrhythmia, the presumed cause for syncope was categorised in ventricular tachyarrhythmia.

\section{Follow-up}

Patients were followed from 1 to 8 years or until heart transplantation (mean $4 \pm 2$ ).

A pacemaker was implanted in patients in whom conduction anomalies were noted at EPS. Patients with induced supraventricular or ventricular tachycardia were treated with an association of amiodarone and beta-blocker. The treatment of ventricular arrhythmias was electrophysiologically guided when LVEF was more than $35 \%$, mainly before 2000 . ICD was usually implanted in patients with still a rapid inducible VT or when LVEF was less than $36 \%$. A specific treatment (percutaneous coronary angioplasty or coronary artery bypass surgery) was indicated in patients with ischaemia. The treatment of ischaemia was also indicated in patients with ischaemia and inducible arrhythmia.

Total cardiac mortality included deaths related to heart failure and sudden deaths. Sudden death was defined as an unexpected death from a cardiac cause within a short time period $(>1 \mathrm{~h}$ ); deaths in relation to the development of a spontaneous sustained VT were classified with sudden deaths. Most deaths occurred in our hospital; for those who died at home or in another hospital, we contacted the last present medical doctor and the family to classify the nature of death.

\section{Statistical analysis}

Quantitative data were expressed as mean \pm SD. Comparisons of patients according to the possible cause of syncope and then according to the follow-up were performed with the Student unpaired $t$ test for quantitative data, with the $\chi^{2}$ test for discrete variables. A p value of $<0.05$ was considered as significant. Univariate analysis by the Cox method and multivariate analysis were performed to identify the independent variables predictive of cardiac and sudden death. Survival curves were calculated using the Kaplan-Meier product-limit method and compared using the logrank test. The predictive negative and positive values of programmed ventricular stimulation data to predict cardiac death were calculated.

\section{RESULTS}

\section{Non-invasive studies}

The mean LVEF was $43 \pm 14 \%$. Holter monitoring was abnormal with ventricular couplets or non-sustained VT in 141 patients $(40 \%)$

The head-up tilt test reproduced syncope in 23 patients who had negative EPS, in four patients in whom a ventricular flutter was induced and in eight with inducible atrial tachyarrhythmias. In other patients, the test remained negative or induced a small decrease in arterial blood pressure without any symptoms. Hypervagotonia was retained as the main diagnosis only if other investigations were negative.

Exercise testing was positive in 54 patients, and non-sustained polymorphic VT developed during exercise in 30 of them. Myocardial ischaemia ( $>40 \%$ ) was noted during the thallium201 exercise scintigraphy and was found to be the only abnormality in 37 patients. Coronary ischaemia was noted in 26 other patients, associated with inducible atrial tachycardia in eight, with inducible VT in 17 and with conduction abnormalities in one patient.

\section{Electrophysiological study}

Sustained monomorphic VT $<270$ bpm (from 150 to 265 bpm, mean $205 \mathrm{bpm}$ ) or syncopal non-sustained VT (frequency from 220 to $240 \mathrm{bpm}$ ) was induced in 87 patients $(24 \%)$, more frequently in patients with low LVEF (37\%) than in those with preserved LVEF $(17 \%)(p<0.001)$. VF was induced in 63 patients (18\%) with the same frequency in patients with low LVEF (19\%) than in those with preserved LVEF (16\%). Ventricular tachyarrhythmia was induced in a control state in 140 patients and after isoproterenol infusion in 10 patients. VT/VF was induced by one extrastimulus in 19 patients, two extrastimuli in 70 patients and three extrastimuli in 61 patients. Seventeen patients had associated coronary ischaemia.

Atrial tachyarrhythmia was the only finding in 39 patients (11\%) (12 paroxysmal junctional tachycardia, 14 atrial tachycardia or flutter, 13 atrial fibrillation). The incidence was similar in patients with low LVEF (8.5\%) and preserved LVEF (13\%). The induction of atrial tachyarrhythmias was noted in association with another abnormality in 13 patients.

Conduction abnormality was the only finding in 23 patients (6\%), seven of these with low LVEF and 16 with preserved LVEF. They were noted in association with VT/VF in 10 other 
Table 1 Main possible causes of syncope

\begin{tabular}{lc}
\hline Ventricular arrhythmia & $150(42 \%)$ \\
Supraventricular tachycardia & $39(11 \%)$ \\
Coronary ischaemia alone & $37(10.4 \%)$ \\
Conduction anomaly & $23(6.5 \%)$ \\
Hypervagotonia & $23(6.5 \%)$ \\
Unexplained & $84(23.6 \%)$ \\
\hline
\end{tabular}

patients, with atrial arrhythmias in five patients and hypervagotonia in three patients.

Carotid sinus massage was positive in 15 patients, but other arrhythmias were noted in these patients, and hypervagotonia was not retained as the cause of syncope.

EPS remained negative in 144 patients, with the same frequency in patients with low LVEF and in those with preserved LVEF.

\section{Possible causes for syncope}

Table 1 summarises the possible causes for syncope.

\section{Analysis of investigations}

Non-invasive and invasive investigations were negative in 84 patients $(23.5 \%)$. Three patients had non-cardiac-related syncope (table 2).

Statistical analysis indicated that patients with conduction abnormalities were older than other patients $(p<0.05)$, and patients with inducible ventricular tachyarrhythmia were more often men $(p<0.01)$.

Table 3 reports the data on non-invasive studies according to the results of the electrophysiological study. Salvos of ventricular beats on Holter monitoring were more frequent in patients with inducible VT than in those without VT/VF. LVEF was lower in patients with inducible VT and VF than in those with negative programmed ventricular stimulation. Patients with inducible VT had a longer ORS duration than patients with inducible VF or negative programmed ventricular stimulation.

Patients with negative studies were more frequently women than patients with inducible ventricular tachyarrhythmia. Table 4 reports the diagnosis value of programmed ventricular stimulation for the identification of a VT as cause for syncope.

\section{Follow-up}

(1 to 6 years, mean $4 \pm 2$ ); 4 patients were lost of view after at least 2 years of follow-up. Patients were followed from 1 to 6 year (mean $4 \pm 2$ ) Fifty-six patients died from a cardiac death; 19 of them died suddenly, and 37 died from heart failure (table 5). Four other patients died from a non-cardiac cause. Patients who died from heart failure were older than remaining patients.
Cardiac defibrillator was implanted in 22 patients. When the patient had multiple positive tests, we attempted to treat each of them.

Global survival was $65 \%$ at 6 years in patients with low LVEF and $80 \%$ in those with preserved LVEF (logrank 18.71; $\mathrm{p}<0.0004)$. Non sustained VTs on Holter monitoring were more frequent in patients who died suddenly (table 4). There was a higher total cardiac mortality in patients with inducible VT and VF than in those with negative electrophysiological study $(p<0.001)$. Induced VT increased the risk of death from heart failure $(p<0.01)$ and sudden death $(p<0.001)$. Cox analysis indicated that inducible VT was more frequent in patients who died from cardiac death $(p<0.001)$, from heart failure $(p<0.02)$ or from sudden death (or who died suddenly) $(p<0.01)$; low LVEF was another predictive factor of total cardiac death $(p<0.0005)$, death by heart failure $(p<0.01)$ or sudden death $(p<0.01)$. Multivariate analysis indicated that induction of VT/ VF and LVEF less than $40 \%$ are predictors of total cardiac mortality (OR respectively 10.2, 7.9); only VT induction was a significant predictor of SD (OR 6.9); advanced age and LVEF $<40 \%$ were significant predictors of death by heart failure (OR 6.3, 5.5) (table 6).

Only two patients with negative non-invasive and invasive studies had recurrences of syncope 18 months and 3 years respectively after the first event. Another complete evaluation including loop recorder implantation remained negative. Loop recorder implantation indicated in 8 other patients with negative studies did not reveal arrhythmias in 7 of them. Another one patient presented a non sustained VT but remained asymptomatic. However, no patient had syncope during recording.

\section{Proposed algorithm to manage patients with syncope and history of myocardial infarction}

The management depends on several factors as the presence of other organic disease, the measurement of LVEF in stable conditions and the results of all investigations including systematic coronary status evaluation (figure 1).

\section{DISCUSSION}

Non-invasive and invasive studies were abnormal in $76 \%$ of patients with syncope and a history of myocardial infarction. In patients with a negative evaluation, the risk of death was low and the recurrence of syncope rare. The study confirms the interest of exercise testing (associated with thallium scintigraphy or dobutamine echocardiography) and head-up tilt testing and the diagnostic value of EPS in postmyocardial infarction ${ }^{12}$ and in patients with syncope. ${ }^{31314}$ Coronary ischaemia was not rare either as the sole factor of syncope or as in association with an arrhythmia. The high mortality in patients with low LVEF

Table 2 Presumed causes of syncope

\begin{tabular}{lllllc}
\hline & & Age & & $\begin{array}{l}\text { Left ventricular } \\
\text { ejection } \\
\text { fraction (\%) }\end{array}$ & Positive Holter M \\
\hline VT/VF & No & (years) & Sex (female) & $76(51 \%)$ \\
Supraventricular tachyarrhythmia & 39 & $66 \pm 11.5$ & $6(15 \%)$ & $45 \pm 12.5$ & $13(33 \%)$ \\
Conduction disturbances & 23 & $72 \pm 11$ & $7(30 \%)^{*}$ & $46 \pm 13$ & $8(35 \%)$ \\
Vagal syncope & 23 & $60 \pm 13.5$ & $3(13 \%)$ & $46 \pm 16$ & $5(22 \%)^{*}$ \\
Ischaemia alone & 37 & $64 \pm 12$ & $3(8 \%)$ & $50 \pm 13^{*}$ & $14(38 \%)$ \\
Unexplained & 84 & $63 \pm 12$ & $23(27 \%)^{* * *}$ & $43 \pm 15$ & $25(30 \%)^{* *}$ \\
\hline
\end{tabular}

In the case of associated abnormalities, induction of a monomorphic ventricular tachycardia/ventricular fibrillation (VT/VF) was considered as the possible cause.

Positive Holter $\mathrm{M}$, presence of salvos of non-sustained VT at $24 \mathrm{~h}$ Holter monitoring.

${ }^{*} \mathrm{p}<0.05 ;{ }^{* *} \mathrm{p}<0.01 ;{ }^{* * *} \mathrm{p}<0.001$ : comparisons with the group VT/NF. 
Table 3 Clinical and electrophysiological data according to the induction of a ventricular tachyarrhythmia

\begin{tabular}{llll}
\hline & $\begin{array}{l}\text { Induced ventricular } \\
\text { tachycardia } \\
(\mathbf{n = 8 7 )}\end{array}$ & $\begin{array}{l}\text { Induced ventricular } \\
\text { fibrillation } \\
(\mathbf{n}=\mathbf{6 3})\end{array}$ & $\begin{array}{l}\text { Negative study } \\
(\mathbf{n}=\mathbf{2 0 6})\end{array}$ \\
\hline $\begin{array}{l}\text { Positive Holter M } \\
\text { Left ventricular ejection }\end{array}$ & $46(53 \%)^{* * *}$ & $30(48 \%)^{*}$ & $65(31.5 \%)$ \\
fraction (\%) & & $41 \pm 15^{* *}$ & $46 \pm 12$ \\
ORS duration (ms) & $139 \pm 31^{* *}$ & $122 \pm 32$ & $115 \pm 28$ \\
Total cardiac death & $30(34 \%)^{* * *}$ & $11(17 \%)$ & $15(7 \%)$ \\
Sudden death & $13(15 \%)^{* * *}$ & $2(3 \%)$ & $4(2 \%)$ \\
Death/heart failure & $17(19.5 \%)^{* *}$ & $9(17 \%)$ & $11(9 \%)$ \\
\hline
\end{tabular}

Positive Holter M, presence of salves of non-sustained ventricular tachycardia under $24 \mathrm{~h}$ Holter monitoring

Comparisons with patients with negative study: ${ }^{* *} \mathrm{p}<0.001 ;{ }^{* *} \mathrm{p}<0.01, \mathrm{p}<0.05$.

and inducible VT was previously reported. ${ }^{72}{ }^{15-17}$ However, programmed ventricular stimulation does not predict sudden death but only ventricular occurrence of arrhythmias. ${ }^{2} 12$

The significance of inducible VF remains debated; the induction is considered without clinical significance in asymptomatic patients in postmyocardial infarction ${ }^{15} 18$ and in patients with syncope and coronary artery disease; in other studies, the VF induction is considered as pathological, but these studies included patients with spontaneous VT. In our study, we note a slightly higher cardiac mortality in patients with decreased LVEF and inducible VF than in patients with a negative study, but deaths were related to heart failure.

Negative EPS in patients with an LVEF less than $40 \%$ indicates a prognosis similar to a population without syncope ${ }^{15} 19$ and a favourable medium-term outcome, principally dependent on the value of LVEF.

Syncope in patients with heart disease has as many possible causes as in patients without heart disease. Bradyarrhythmias are not rare. ${ }^{20}$ Vagal hypertonia could be a frequent cause of syncope. ${ }^{21}$ Supraventricular tachyarrhythmia is reported in about $10 \%$ of the patients with syncope and heart disease. ${ }^{20} 22$ This last cause could be a frequent cause of syncope but remains difficult to prove: salvos of atrial premature beats on Holter monitoring are frequent and not specific. In patients with low LVEF, rapid supraventricular tachyarrhythmia induces a decrease in cardiac output. In patients with preserved LVEF, the increase in vagal tone in answer to the tachycardia could explain syncope, when tachycardia stops. ${ }^{22}$ Similar mechanism could explain why a ventricular monomorphic VT can induce syncope when LVEF is preserved; previous studies showed that a VT or a rapid ventricular pacing induces an adrenergic tone increase followed by a secondary vagal hypertonia.

Another facilitating mechanism for syncope associated with supraventricular or ventricular tachycardia was the presence of coronary ischaemia. ${ }^{23}$ Syncope is usually exercise-related and might be related to an ischaemic non-sustained polymorphic VT and to a decrease in cardiac output when a narrowing of main left coronary artery is present. Several studies have shown that anti-ischaemic treatment did not prevent the recurrences of tachycardia but improved their tolerance. ${ }^{23}{ }^{24}$ Moreover,

Table 4 Diagnostic value of programmed ventricular stimulation

\begin{tabular}{lllllll}
\hline & PPV & PPV & PPV & NPV & NPV & NPV \\
& CD & SD & HF & CD & SD & HF \\
Ventricular tachycardia/ventricular & $82.5 \%$ & $88 \%$ & $78 \%$ & $95 \%$ & $98.5 \%$ & $96 \%$ \\
flutter/fibrillation & & & & & &
\end{tabular}

$\mathrm{CD}$, total cardiac death; HF, death related to heart failure; NPV, negative predictive value; PPV, positive predictive value; SD, sudden death.
Table 5 Clinical and electrophysiological data of total population according to the follow-up

\begin{tabular}{|c|c|c|c|c|}
\hline & $\begin{array}{l}\text { Death/heart } \\
\text { failure }\end{array}$ & $\begin{array}{l}\text { Sudden } \\
\text { death }\end{array}$ & $\begin{array}{l}\text { Total cardiac } \\
\text { death }\end{array}$ & Alive \\
\hline No & 37 & 19 & 56 & 300 \\
\hline Age (years) & $72 \pm 8.5^{* * *}$ & $65 \pm 10$ & $69 \pm 9.5$ & $65 \pm 11.5$ \\
\hline Female sex & $9(24 \%)$ & $2(10.5 \%)$ & $11(20 \%)$ & $45(15 \%)$ \\
\hline Positive Holter M & $13(35 \%)$ & $13(68 \%)^{*}$ & $26(47 \%)$ & $115(30 \%)$ \\
\hline $\begin{array}{l}\text { Left ventricular ejection } \\
\text { fraction }(\%)\end{array}$ & $33 \pm 12$ & $31 \pm 12$ & $33 \pm 12$ & $43 \pm 4^{* * *}$ \\
\hline Induced VT/VF & $25(67.5 \%)^{* * * *}$ & $\begin{array}{l}15(79 \%) \\
* * *\end{array}$ & $40(73 \%)^{* * *}$ & $109(36 \%)$ \\
\hline Induced VT & $17(46 \%)^{* *}$ & $\begin{array}{l}13(68 \%) \\
* *\end{array}$ & $30(54.5 \%)^{* * *}$ & $57(19 \%)$ \\
\hline Induced VF & $9(24 \%)$ & $2(10.5 \%)$ & $11(20 \%)$ & $52(17 \%)$ \\
\hline Negative study & $11(30 \%)^{* * *}$ & $\begin{array}{l}4(21 \%) \\
* * *\end{array}$ & $15(27 \%)^{* * *}$ & $191(64 \%)$ \\
\hline
\end{tabular}

Positive Holter M, presence of salves of non-sustained ventricular tachycardia under $24 \mathrm{~h}$ Holter monitoring; VF, ventricular flutter/fibrillation; VT, monomorphic ventricular tachycardia $<270 \mathrm{bpm}$

${ }^{*} \mathrm{p}<0.05 ;{ }^{* *} \mathrm{p}<0.01 ;{ }^{* *} \mathrm{p}<0.001$

ischaemia is an independent predictor of death, and Elhendy et al $^{23}$ reported that the combination of ischaemia and a positive EPS is associated with a very high rate of events. Shaw et a ${ }^{25}$ recommended an aggressive drug evaluation by serial myocardial perfusion single photon emission computer tomography to enhance the prognosis. ${ }^{25}$ Therefore, we recommend a systematic evaluation of the coronary status in the case of unexplained syncope occurrence in patients with a history of coronary heart disease, even in the absence of symptoms such as exercise-related syncope or syncope associated with chest pain. Myocardial ischaemia was noted in $15 \%$ of our population, and in $10 \%$ of this population, ischaemia was the only cause for syncope. The treatment by coronary angioplasty or coronary bypass surgery of coronary narrowing suppressed the recurrence of syncope.

\section{LIMITATIONS OF THE STUDY}

The role of myocardial ischaemia alone or associated with a tachyarrhythmia could have been underestimated in the present study, because thallium exercise scintigraphy was not systematic, and only an exercise test was performed in 240 patients.

The incidence of neurocardiogenic syncope may also have been underestimated because the tilt test was not systematic. A patient may have supraventricular or ventricular tachycardia and also enhanced vagal tone.

Table 6 Stepwise logistic regression analysis to identify the independent variables predictive of cardiac death, sudden death and death by heart failure

\begin{tabular}{llll}
\hline Predictive factors & OR & $\mathbf{p}$ Value & $\mathbf{9 5 \%} \mathbf{C l}$ \\
\hline Cardiac death & & & \\
$\quad$ VT & 10.1644 & 0.0014 & 1.886 to 14.282 \\
VF & 1.072 & 0.36 & 0.533 to 7.609 \\
$\quad$ LVEF $<40$ versus $>40 \%$ & 9.6100 & 0.0019 & 1.474 to 8.917 \\
Sudden death & & & \\
$\quad$ VT & 6.9518 & 0.0084 & 2.050 to 131.037 \\
$\quad$ LVEF $<40$ versus LVEF $>40 \%$ & 3.7333 & 0.0533 & 0.971 to 58.938 \\
$\begin{array}{l}\text { Death by heart failure } \\
\quad \text { Age }\end{array}$ & 6.3286 & 0.0119 & 1.019 to 1.168 \\
$\quad$ LVEF $<40$ versus $>40 \%$ & 5.5173 & 0.0188 & 1.503 to 91.392
\end{tabular}

LVEF, left ventricular ejection fraction; VF, ventricular flutter/fibrillation; VT, ventricular tachycardia. 
Clinical history

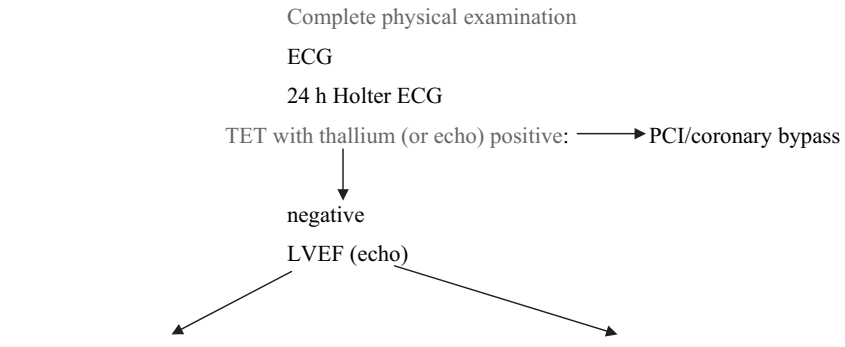

LVEF $<30 / 36 \%$

Good expectation of life

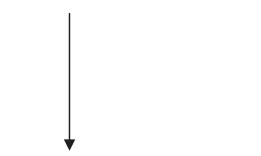

EPS: specific treatment $+\mathrm{ICD}$

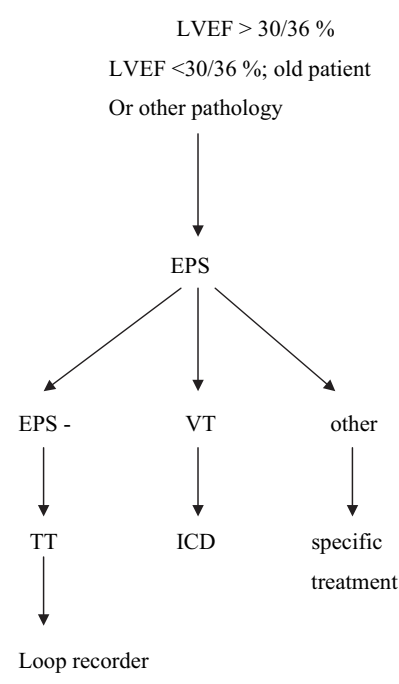

Figure 1 Proposed diagram for the management of patients with syncope and history of myocardial infarction. -, negative; EPS, electrophysiological study; ICD, implantable cardiac defibrillator; LVEF, left ventricular ejection fraction; Other, abnomaly of electrophysiological study except inducible ventricular tachycardia; $\mathrm{PCI}$, percutaneous coronary intervention; TET, Treadmill exercise testing; TT, tilt test; VT, inducible ventricular tachycardia.

The changes in treatments during the 15 years of recruitment could have modified the prognosis of our patients. However, a previous study published in $2003^{26}$ reported the data of patients recruited earlier, and the prognosis was similar.

EPS was performed in a supine position, and only patients with a rapid non-sustained monomorphic VT reproduced their syncope. In those with inducible supraventricular tachycardia, only dizziness or a drop in arterial blood pressure was noted.

Data from the loop recorder implantation were not reported because the system was implanted in only eight patients with negative EPS.

The exact value of LVEF considered as pathological has changed during the recruitment, but we know also that values between 30 and $40 \%$ are frequently variable, and the indications of ICD varied from 30 to $40 \%$, depending on the NYHA stage, the presence of NS VT at Holter monitoring and several other factors.

In conclusion, several causes such as coronary ischaemia, neurocardiogenic origin or supraventricular tachyarrhythmia might be implicated in the mechanism of syncope after myocardial infarction. The evaluation of coronary status is recommended; silent myocardial ischaemia was probably an underestimated facilitating cause of syncope. Thus, complete EPS remains a rapid tool to identify atrioventricular block, supraventricular or ventricular tachyarrhythmia. Induced monomorphic VT was the best predictor of sudden death. Advanced age and low LVEF were predictors of death by heart failure. Patients with unexplained syncope were at low risk of event.
Competing interests None.

Provenance and peer review Not commissioned; externally peer reviewed.

\section{REFERENCES}

1. Moss AJ, Zareba W, Hall J, et al. For the multicenter automatic defibrillator implantation. Trial II investigators. Prophylactic implantation of a defibrillator in patients with myocardial infarction and reduced ejection fraction. $N$ Engl J Med 2002; 346:877-83.

2. Kapoor WN. Current evaluation and management of syncope. Circulation 2002;106:1606-9.

3. Mittal S, Iwai S, Stein KM, et al. Long-term outcome of patients with unexplained syncope treated with an electrophysiologic-guided approach in the implantable cardioverter-defibrillator era. J Am Coll Cardiol 1999:34:1082-9.

4. Krol R, Morady F, Flaker C, et al. Electrophysiologic testing in patients with unexplained syncope: clinical and noninvasive predictors of outcome. J Am Coll Cardiol 1987:10:358-63.

5. Moya A, Sutton R, Ammirati F, et al. Developed in collaboration with, European Heart Rhythm Association (EHRA); Heart Failure Association (HFA); and Heart Rhythm Society (HRS); Guidelines for the diagnosis and management of syncope (version 2009): the task force for the diagnosis and management of syncope of the European Society of Cardiology (ESC). Eur Heart J 2009;30:2631-71

6. Daubert JP, Zareba W, Hall WJ, et al. MADIT II Study Investigators. Predictive value of ventricular arrhythmia inducibility for subsequent ventricular tachycardia or ventricular fibrillation in Multicenter Automatic Defibrillator Implantation Trial (MADIT) II patients. J Am Coll Cardiol 2006;47:108-11.

7. Buxton $\mathbf{A E}$, Lee $\mathrm{KL}$, Hafley $\mathrm{GE}$, et al. Limitations of ejection fraction for prediction of sudden death risk in patients with coronary artery disease. J Am Coll Cardiol 2007; 50:1150-7.

8. Arya A, Haghjoo M, Dehghani R, et al. Prevalence and predictors of electrical storm in patients with implantable cardioverter-defibrillator. Am J Cardiol 2006;97:389-92

9. Krahn AD, Klein GJ, Yee R, et al. Final results from a pilot study with implantable loop recorder to determine the etiology of syncope in patients with negative noninvasive and invasive testing. Am J Cardiol 1998;82:117-19.

10. Boersma L, Mont L, Sionis A, et al. Value of the implantable loop recorder for the management of patients with unexplained syncope. Europace 2004;6:70-6.

11. Farwell DJ, Freemantle N, Sulke AN. Use of implantable loop recorders in the diagnosis and management of syncope. Eur Heart J 2004;25:1257-63.

12. Brembilla-Perrot B, Suty-Selton C, Beurrier D, et al. Differences in mechanisms and outcomes of syncope in patients with coronary disease or idiopathic left ventricular dysfunction as assessed by electrophysiologic testing. J Am Coll Cardiol 2004;44:594-601.

13. Andrews NP, Fogel RJ, Pelargonio G, et al. Implantable defibrillator event rates in patients with unexplained syncope and inducible sustained ventricular tachyarrhythmias. J Am Coll Cardiol 1999;34:2023-30.

14. Mont L, Valentino M, Sambola A, et al. Arrhythmia recurrence in patients with a healed myocardial infarction who received an implantable defibrillator: analysis according to the clinical presentation. J Am Coll Cardiol 1999:34:351-7.

15. Pezawas T, Stix G, Kastner J, et al. Unexplained syncope in patients with structura heart disease and no documented ventricular arrhythmias: value of electrophysiologically guided implantable cardioverter defibrillator therapy. Europace 2003:5:305-12.

16. Buxton AE, Lee $\mathrm{KL}$, Hafley GE, et al. For the MUSTT investigators. Relation of ejection fraction and inducible ventricular tachycardia to mode of death in patients with coronary artery disease. An analysis of patients enrolled in the multicenter unsustained tachycardia trial. Circulation 2002;106:2466-72.

17. Letorier $\mathbf{P}$, Krahn AD, Lein GJ, et al. Comparison of patients with syncope with left ventricular dysfunction and negative electrophysiologic testing to cardiac arrest survivors and patients with syncope and preserved left ventricular function and impact of an implantable defibrillator. J Am Coll Cardiol 2002;78:77-9.

18. Brembilla-Perrot B, Suty-Selton C, Claudon 0, et al. Significance of inducible ventricular flutter-fibrillation after myocardial infarction. PACE 2005;28:938-43.

19. Menozzi C, Brignole M, Garcia-Civera R, et al. on behalf of the International Study on Syncope of Uncertain Etiology. (ISSUE) Investigators. Mechanism of syncope in patients with heart disease and negative electrophysiologic test. Circulation 2002; 105:2741-5.

20. Link MS, Kim KMS, Homoud MK, et al. Long-term outcome of patients with syncope associated with coronary artery disease and a nondiagnostic electrophysiologic evaluation. Am J Cardiol 1999:83:134-7.

21. Alboni $\mathbf{P}$, Brignole $\mathrm{M}$, Menozzi $\mathrm{C}$, et al. Diagnostic value of history in patients with syncope with or without heart disease. J Am Coll Cardiol 2001;37:1921-8.

22. Brembilla-Perrot B, Beurrier D, Houriez P, et al. Incidence and mechanism of presyncope and/or syncope associated with paroxysmal junctional tachycardia. Am J Cardiol 2001:88:134-8.

23. Elhendy A, Chapman S, Porter TR, et al. Association of myocardial ischaemia with mortality and implantable cardioverter- defibrillator therapy in patients with coronary artery disease at risk of arrhythmic death. J Am Coll Cardiol 2005; 46:1721-6 
24. Brugada J, Aguinaga L, Mont L, et al. Coronary artery revascularization in patients with sustained ventricular arrhythmias in the chronic phase of a myocardial infarction: effects on the electrophysiologic substrate and outcome. J Am Coll Cardiol 2001:37:529-33.

25. Shaw LJ, Berman DS, Maron DJ, et al. Courage investigators. Optimal medical therapy with or without percutaneous coronary intervention to reduce ischaemic burden: results from the Clinical Outcomes Utilising Revascularization and Aggressive Drug Evaluation (COURAGE) trial nuclear substudy. Circulation 2008;117:1283-91.

26. Brembilla-Perrot B, Suty-Selton C, Alla F, et al. Facteurs de risque de mortalité cardiaque en cas de syncope et d'antécédents d'infarctus du myocarde. (In French). Arch Mal Cœur 2003;96:1181-6. 